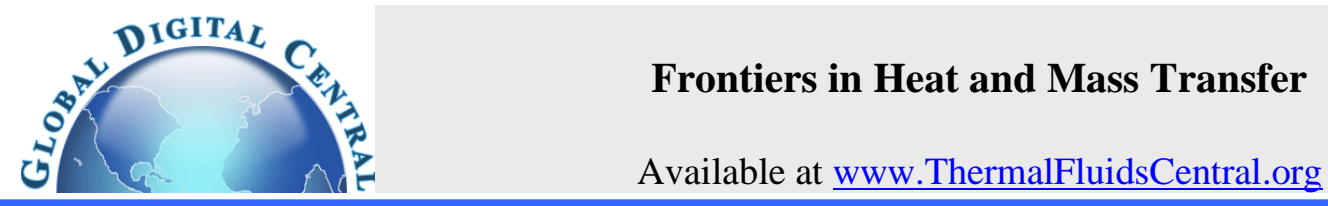

\title{
WATER TRANSPORT IN CELLULAR CONNEXON OF HUMAN BODIES
}

\author{
Mian Wang ${ }^{1}$ and Yongbin Zhang ${ }^{2 *}$ \\ 1. School of Electronic Engineering, Changzhou College of Information Technology, Changzhou, 213164, Jiangsu Province, China \\ 2. College of Mechanical Engineering, Changzhou University, Changzhou, 213164, Jiangsu Province, China
}

\begin{abstract}
There is the connexon between neighboring cells in human bodies, which normally has the cylindrical channels with the diameter about $1.5 \mathrm{~nm}$. The analysis is here derived for the water transport through such a narrow channel based on the nanoscale flow equation by considering the dynamic, interfacial slippage and non-continuum effects of the water. The calculation shows that when the intracellular fluids of the neighboring cells are not obviously different, there is no wall slippage in the connexon channel and the water flow rate through the channel is significantly smaller than that calculated from the classical continuum flow equation due to the viscous and non-continuum effects of the water; However, when the intracellular fluids of the neighboring cells are much different, there would be the intracellular pressure difference between the neighboring cells which is large enough for starting the wall slippage in the channel; For the wall slippage case, the water transport rate through the channel is normally much greater than and can be 1 to 7 orders larger than the classical flow equation calculation, just only by the power losses ranging between $1.0 \mathrm{E}-14 \mathrm{Watt}$ and $1.0 \mathrm{E}-$ 9Watt on each channel. This transport characteristic is very important for quickly achieving the balance between the neighboring cells by fast transporting the water through the connexon channel.
\end{abstract}

Keywords: Cellular connexon; Flow; Interfacial slippage; Nanochannel

\section{INTRODUCTION}

Healthy cells play important roles in maintaining the lives of animals including human beings. They exchange the information and substances with each other and with interstitial fluids. To understand the mechanism of the operation of cells is very important for medical treatment of human beings. There is the connexon between the neighboring cells, which normally has the cylindrical channels with the diameter about $1.5 \mathrm{~nm}$ for transporting water and other small substances. This function is very important for maintaining the ordinary harmonic actions of the cells close to each other. The diameter of the cellular connexon channel is reduced with the increase of the value of $\mathrm{PH}$ in the cell. If there is a significant difference between the intracellular fluids of the neighboring cells, water and other small substances will be fast transported from one cell to the other cell through the channel to maintain nearly the same physiological level of the neighboring cells. If the intracellular fluids of the neighboring cells are nearly the same, there will be only a little exchange between the two cells through the channel. If one cell is dying, its connexon channel may be just closed to avoid any exchange with the neighboring cells. To understand how the water is transported through the cellular connexon channel is of significant interest to the medical knowledge and curing.

The flow regime of water in a channel may be continuum, multiscale or non-continuum depending on the channel height ( $\mathrm{Li}, 2010$; Walther et al., 2012; Whitby et al., 2008). It was suggested (Zhang, 2020; $\mathrm{Li}$ and Zhang, 2021) that when the ratio $\lambda_{b f}$ of the total thickness of the adsorbed layers on the channel walls to the channel height is over 100 , the continuum flow theory is valid; When $1<\lambda_{b f}<100$, the liquid flow regime should be multiscale owing to the adsorbed layer effect; When $\lambda_{b f}<1$, the flow should be non-continuum owing to the adsorbed layers entirely filling the channel height.

Even for the water in the hydrophobic carbon nanotube with the diameter 2.0nm, the water flow was believed to be non-continuum (Borg and Reese, 2017). For such a narrow channel flow, the classical continuum flow theory is invalid. It was found by experiments that the water flow rate through the carbon nanotube with the diameter no more than $7 \mathrm{~nm}$ is much larger than and can be $4-5$ orders higher than that calculated from the classical Hagen-Poiseuille equation (Borg and Reese, 2017; Holt et al., 2006; Majumder et al., 2005). There have been several mechanisms proposed for explaining this unexpected high flow rate such as the depletion layer effect and the interfacial slippage effect.

The water flow in the cellular connexon channel should be noncontinuum. Molecular dynamics simulation can model this water flow because of the small dimensions of the channel. In the present study, we used the closed-form nanoscale flow equation (Zhang, 2016), which has been validated (e.g. see the recent work by Jiang and Zhang (2021)), to calculate the water flow rate through this narrow channel. Detailed analytical results have been derived respectively for the cases of wall slippage and no wall slippage. Important calculation results were obtained indicating the valuable information for the operation of cells.

\section{WATER FLOW IN A CELLULAR CONNEXON CHANNEL}

Figure 1 shows the connexon between the neighboring cells in human bodies, which has the cylindrical nanochannel normally with the diameter around $1.5 \mathrm{~nm}$ for transporting water and other very small 
substances, to keep the two cells to react harmonically to the environment. The primary function of this channel is to transport water. Figures 2(a) and (b) show the profiles of such a narrow channel. The axial length $l$ of the channel is normally between $16 \mathrm{~nm}$ and $19 \mathrm{~nm}$, and the diameter of the channel can be changed by the value of PH and the concentration of $\mathrm{Ca}^{2+}$ inside the cell.

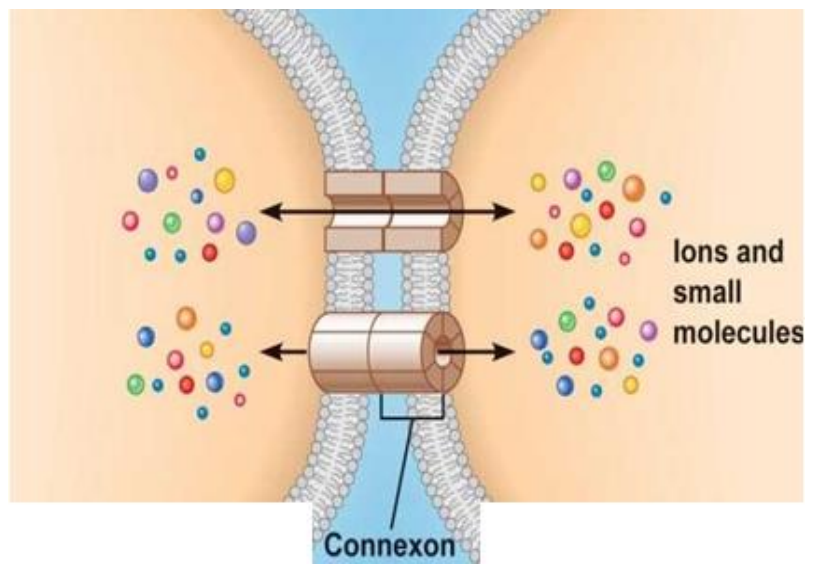

Fig. 1 Connexons between two neighboring cells in human bodies.

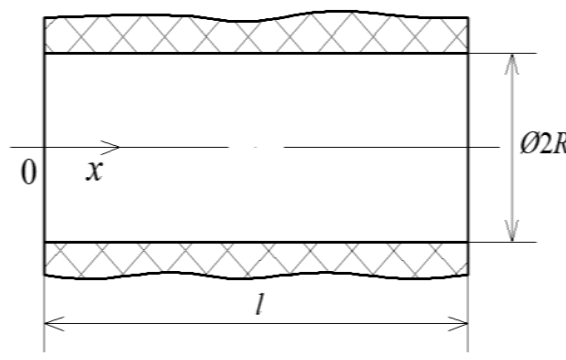

(a)

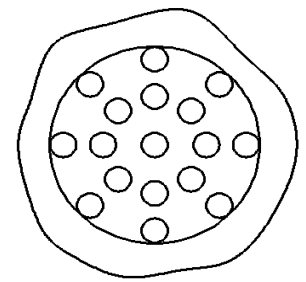

(b)
Fig. 2 A cylindrical nanochannel in the cellular connexon. (a) Front view, $l=16 \sim 19 \mathrm{~nm}, R \approx 0.75 \mathrm{~nm}$; (b) Left-side view, water molecules inside the channel.

\section{ANALYSIS}

In the past, molecular dynamics simulation was popularly used to simulate the water flow in the nanochannels with tiny zones (Khademi and Sahimi, 2011; Thomas et al., 2010; Wang et al., 2012). For the present specific water flow simulation, this method should also be usable. For a quick solution and a good understanding of the water flow characteristic, the present study used the nanoscale flow equation (Zhang, 2016) to calculate the water flow rate through the channel; The influences of the pressure on both the viscosity and density of the water are negligible, and the flow was treated as isothermal.

\subsection{For the case of no wall slippage}

When the two neighboring cells have the very similar intracellular fluids, there is little necessity of the exchange between them. For this case, the difference between the pressures inside these two cells is quite small, and no wall slippage occurs in the connexon channel. In this case, the mass flow rate of the water through the channel should be small, and it is equated as (Zhang, 2016):

$q_{m}=\frac{\pi \rho_{b f}^{e f f}(\bar{R}) S(\bar{R}) R^{4}}{4 \eta_{b f}^{e f f}(\bar{R})} \frac{\partial p}{\partial x}$

where $p$ is the pressure, $R$ is the inner radius of the channel, $x$ is the coordinate in the flow direction, $\rho_{b f}^{e f f}$ is the average density of the water across the channel radius, $\eta_{b f}^{e f f}$ is the effective viscosity of the water in the channel, and $S$ is the parameter describing the non-continuum effect of the flowing water.

The ratio of the value of $q_{m}$ in Eq.(1) to the mass flow rate calculated from the classical continuum flow equation is:

$r_{m}=-\frac{C q(\bar{R}) S(\bar{R})}{\operatorname{Cy}(\bar{R})}$

where $C_{y}=\eta_{b f}^{\text {eff }} / \eta, C_{q}=\rho_{b f}^{\text {eff }} / \rho, \eta$ and $\rho$ are respectively the water bulk viscosity and bulk density.

\subsection{For the case of wall slippage}

When there is a significant difference between the intracellular fluids of the neighboring cells, the pressure drop on the connexon channel will be large enough to motivate the wall slippage in the channel. For this case, the mass flow rate of the water through the channel is (Zhang, 2016):

$q_{m}=\pi \rho_{b f}^{e f f}(\bar{R}) u_{s} R^{2}+\frac{\pi \rho_{b f}^{e f f}(\bar{R}) S(\bar{R}) R^{4}}{4 \eta_{b f}^{\text {eff }}(\bar{R})} \frac{\partial p}{\partial x}$

where $u_{s}$ is the wall slipping velocity.

\subsubsection{Critical power loss on the channel for wall slippage and formulation of wall slipping velocity}

If a liquid is continuum in a cylindrical tube, its flow velocity in the tube is expressed as (Zhang, 2017):

$u(r)=u_{s}+\frac{r^{2}-R^{2}}{2 \eta} \frac{\partial p}{\partial x}$

where $r$ is the radius of the location and $\eta$ is the liquid bulk viscosity. Assume the continuum liquid as Newtonian, the shear stress of the liquid film in the tube is:

$\tau=\eta \frac{\partial u}{\partial r}=r \frac{\partial p}{\partial x}$

For the present case, assume that $\partial p / \partial x<0$, when the wall slippage occurs, the wall shear stress is equal to the water-channel wall interfacial shear strength $\tau_{s}$, and there is:

$-\theta_{\tau}(R) R \frac{\partial p}{\partial x}=\tau_{s}$

where $\theta_{\tau}(R)$ is the correction factor for the shear stress on the wall owing to the non-continuum effect of the water (Zhang, 2006).

Thus, in the present flow with the wall slippage, the dimensional pressure drop on the whole connexon channel is:

$D P=\frac{\tau_{s} l}{\theta_{\tau}(R) R}$

For starting the wall slippage, the difference $(D P)$ between the pressures inside the neighboring cells must reach the level formulated by Eq.(7).

The power loss on the whole channel is:

$P O W=\frac{D P \cdot q_{m}}{\rho_{b f}^{e f f}}=\frac{\tau_{s} l}{\theta_{\tau}(R) R}\left[\pi u_{s} R^{2}-\frac{\pi S(\bar{R}) \tau_{s} R^{4}}{4 \theta_{\tau}(R) R \eta_{b f}^{\text {eff }}(\bar{R})}\right]$

Let $u_{s}=0$, the critical power loss on the channel for initiating the wall slippage is found from Eq.(8) to be:

$P O W_{c r}=-\left(\frac{\tau_{s}}{\theta_{\tau}(R)}\right)^{2} \frac{\pi S(\bar{R}) R^{2} l}{4 \eta_{b f}^{e f f}(\bar{R})}$

Define $D P O W=P O W-P O W_{c r}$, then it is obtained that:

$u_{s}=\frac{D P O W \theta_{\tau}(R)}{\pi \tau_{s} l R}$

Equation (10) shows that in the present channel flow, the wall slipping velocity $u_{s}$ is in direct proportion to $D P O W$.

By substituting Eq.(10) into Eq.(3), the ratio of the value of $q_{m}$ in Eq.(3) to the mass flow rate calculated from the classical HagenPoiseuille equation is:

$r_{m}=\frac{4 C q(\bar{R}) \theta_{\tau}^{2}(R) \eta(D P O W)}{\pi R^{2} \tau_{s}^{2} l}-\frac{C q(\bar{R}) S(\bar{R})}{C y(\bar{R})}$ 


\section{CALCUlations}

In the present calculation, the axial length of the connexon channel was chosen as $17 \mathrm{~nm}$, and the radius $R$ of the channel was varied from $0.3 \mathrm{~nm}$ to $1.0 \mathrm{~nm}$. The other parameter values were chosen as: $\eta=0.001 \mathrm{~Pa}$. s and $\tau_{s}=10 \mathrm{kPa}$. The value of $\theta_{\tau}(R)$ is below 1.0 , and for the present relatively weak interaction of the water with the channel wall it should be close to unity (Zhang, 2006). Here, it was taken that $\theta_{\tau}(R)=1.0$.

Owing to the adsorption of the water film to the channel wall, it is formulated that (Zhang, 2014):

$$
\begin{array}{r}
C y(\bar{R})=0.9507+\frac{0.0492}{\bar{R}}+\frac{1.6447 E-4}{\bar{R}^{2}}, \text { for } 0.1<\bar{R}<1.0 \\
C q(\bar{R})=1.116-0.328 \bar{R}+0.253 \bar{R}^{2}-0.041 \bar{R}^{3}, \\
\text { for } 0.1<\bar{R}<1.0
\end{array}
$$

and

$S(\bar{R})=\left[-0.1-0.892(\bar{R}-0.1)^{-0.084}\right]^{-1}, \quad$ for $0.1<\bar{R}<1.0$ (14) where $\bar{R}=R / R_{c r, b f}$. Here, $R_{c r, b f}$ is the critical radius and chosen as $2.8 \mathrm{~nm}$.

\section{RESULTS}

Figure 3 shows the values of the dimensional pressure drop $(D P)$ on the whole connexon channel calculated from Eq.(7), which are required for the wall slippage occurrence. The values of $D P$ are on the scale $100 \mathrm{kPa}$, rapidly increased with the reduction of the channel diameter $R$. To achieve this $D P$ level, there should be a remarkable difference between the intracellular fluids of the neighboring cells. Once the value of $D P$ is reached, the wall slippage will occur and the water with unexpected high flow rates (Borg and Reese, 2017; Holt et al., 2006; Majumder et al., 2005 ) will come from one cell to the other cell to quickly find the balance of the intracellular environment of the two cells.

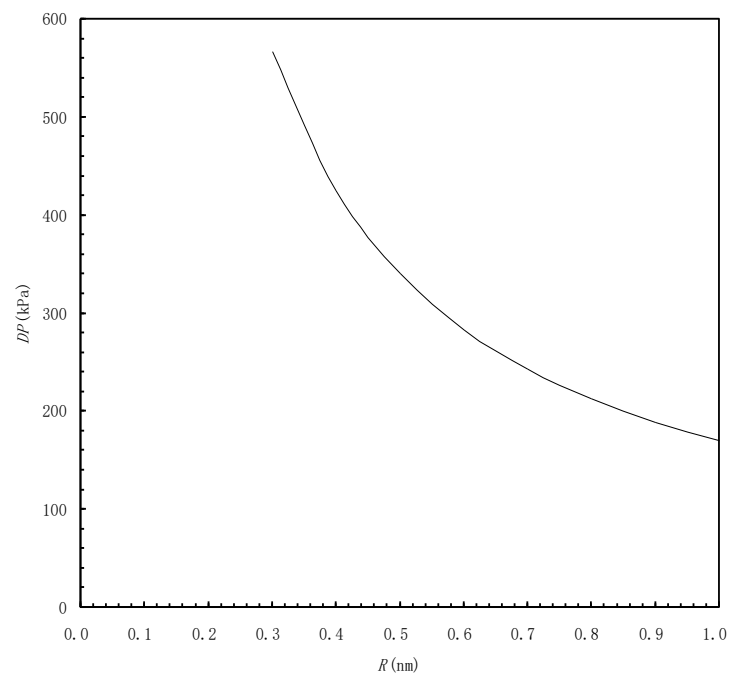

Fig. 3 Pressure drop $(D P)$ on the whole connexon channel required for the wall slippage.

Figure 4 shows the values of the critical power loss $\left(P O W_{c r}\right)$ on the whole connexon channel for initiating the wall slippage. For $R=0.3 \mathrm{~nm} \sim 1.0 \mathrm{~nm}$, the values of $P O W_{c r}$ are on the scales of $1.0 \mathrm{E}-17 \mathrm{Watt}$ to $1.0 \mathrm{E}-15 \mathrm{Watt}$, which are fairly low, and rapidly reduced with the reduction of $R$. These show that the power loss on each connexon channel for initiating the wall slippage is very small and smaller the value of $R$, easier the wall slippage occurrence. It can also be recognized that if the wall slippage is absent, the power loss on the connexon channel is smaller than $P O W_{c r}$, and very small for the transport of slow water between the two cells. This should be the ordinary situation of normal cells.

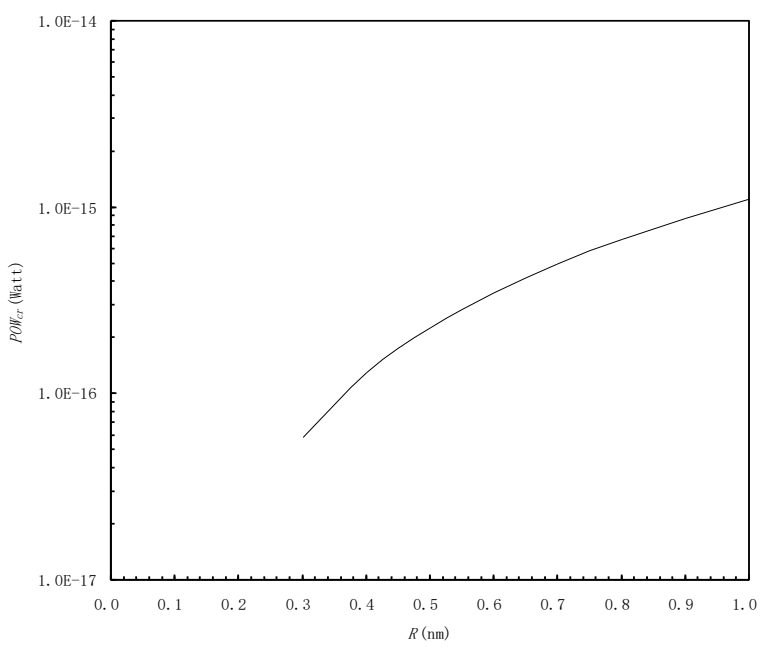

Fig. 4 Critical power loss on the whole connexon channel for initiating the wall slippage.

Figure 5 shows that for a given $D P O W$, the wall slipping velocity $u_{s}$ is significantly increased with the reduction of $R$. It again indicates that smaller the value of $R$, larger the wall slippage. This correlates well with the experimental findings that the water flow rate in the carbon nanotube with the diameter no more than $2.0 \mathrm{~nm}$ is much larger than the classical hydrodynamic theory calculation, attributed to the large wall slippage (Borg and Reese, 2017; Holt et al., 2006; Majumder et al., 2005). On the cell scale, the plotted values of $u_{s}$ in Fig.5 should be large, but just with very small values of $D P O W$. This suggests that in the cell connexon, the significant wall slippage is easily generated just by a very small power loss on each channel.

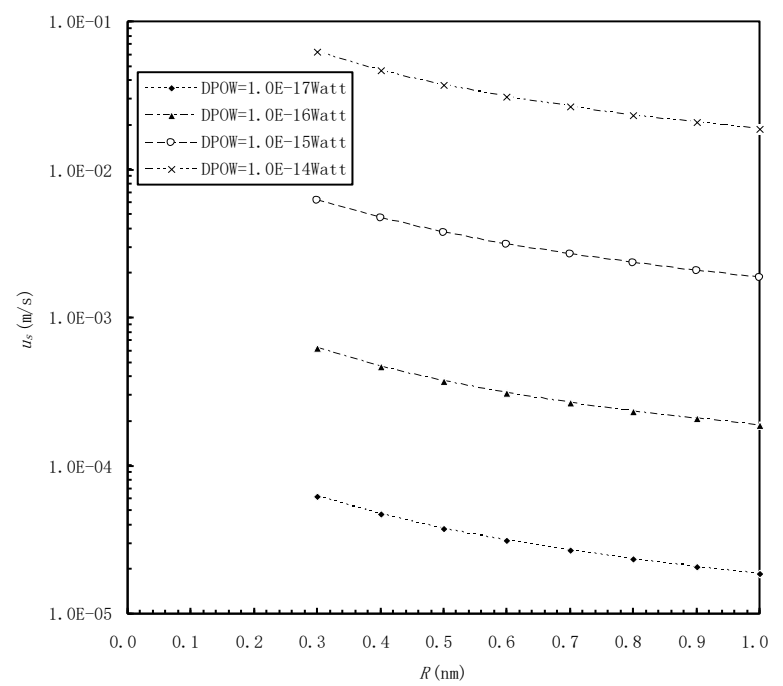

Fig. 5 Wall slipping velocity in the connexon channel as function of the channel diameter $R$ and the power loss difference DPOW.

Figures 6(a) and (b) show the values of $r_{m}$, which is the ratio of the water flow rate through the channel calculated from the non-continuum flow theory to that calculated from the classical Hagen-Poiseuille equation. It is shown that for no wall slippage or the small wall slippage (corresponding to $D P O W=1.0 \mathrm{E}-17 \mathrm{Watt}$ ), the value of $r_{m}$ is significantly reduced with the reduction of $R$ and considerably smaller than unity; This shows that the non-continuum and dynamic effects of the water in such narrow channels result in the much smaller water flow rate than the classical flow theory prediction, and smaller the channel diameter, more significant this combined effect. However, when $D P O W$ is increased to no less than $1.0 \mathrm{E}-16 \mathrm{~W}$ att, the wall slippage is shown to be sufficiently 
large and the wall slippage effect is predominant. For $D P O W>1.0 \mathrm{E}$ 16 Watt, the value of $r_{m}$ is otherwise significantly increased with the reduction of $R$ and obviously greater than unity; This is surely due to the wall slippage effect. The increase of $D P O W$ has a very strong effect in increasing $r_{m}$. For $D P O W=1.0 \mathrm{E}-13 \mathrm{Watt} \sim 1.0 \mathrm{E}-9 \mathrm{~W}$ att, the calculated water mass flow rates are 2 to 6 orders larger than the classical hydrodynamic flow theory calculation, qualitatively agreeing with the experimental finding for the water flow in the carbon nanotubes with the diameter no more than 7.0nm (Borg and Reese, 2017; Holt et al., 2006; Majumder et al., 2005). It is shown that in the cell connexon, the large wall slippage easily occurs and the water flow rate will consequently be far greater than the classical hydrodynamic theory estimation, only with a very small power loss on the connexon channel. This should be the case when there is a significant difference between the two neighboring cells and there is an urgent necessity to find the balance between these two cells. In such a case, the energy loss in the cell will be increased.

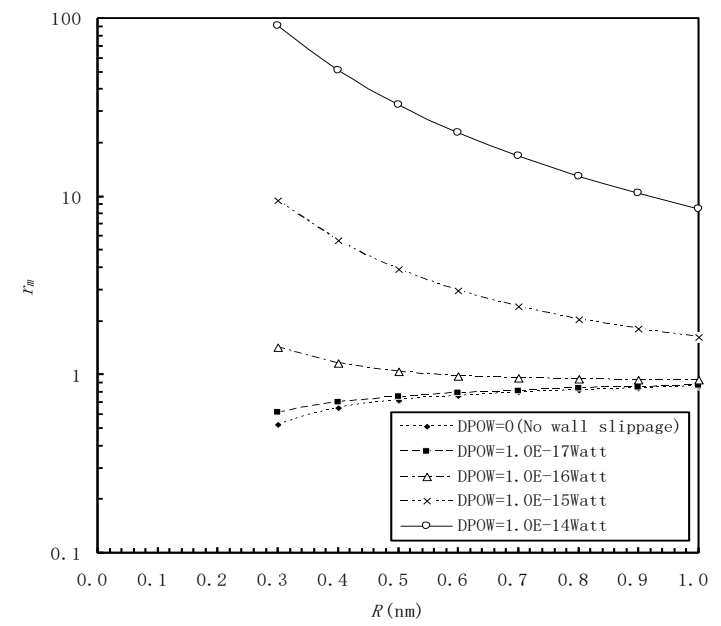

(a)

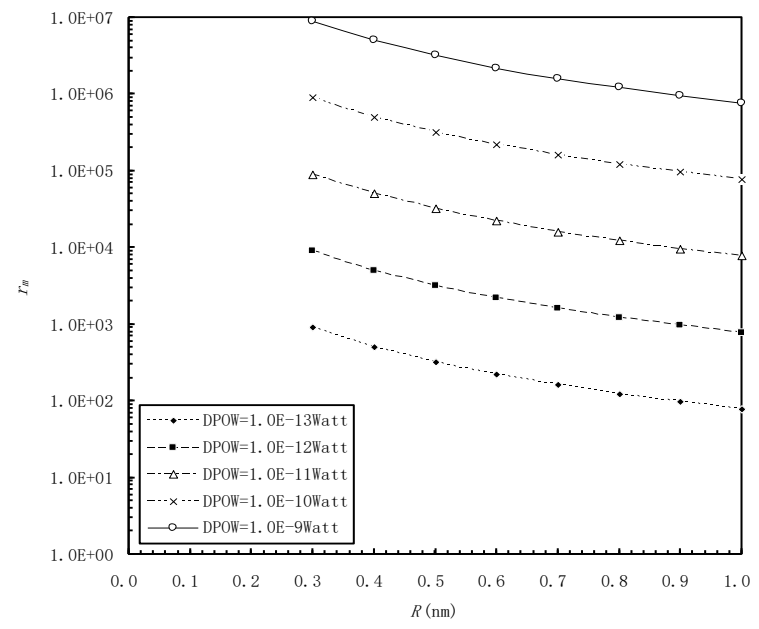

(b)

Fig. 6 Values of $r_{m}$.

\section{CONCLUSIONS}

The analytical derivation was made for the mass flow rate of water through the human cellular connexon channel normally with the diameter around $1.5 \mathrm{~nm}$, based on the nanoscale flow equation (Zhang, 2016). The calculation results show that when the intracellular fluids of two neighboring cells are very similar with one another, there is only a little exchange between these two cells through the channel, no wall slippage occurs in the channel, and the water flow rate through the channel is rapidly reduced with the reduction of the channel diameter $R$ and much smaller than the classical continuum flow equation calculation, owing to the non-continuum and dynamic effects of the water; When there is a significant difference between the intracellular fluids of the two neighboring cells, there is an urgent necessity to find the balance between the two cells to achieve the very similar intracellular fluids of them, the large wall slippage thus occurs in the channel, the resulting water flow rate through the channel is far greater than the classical hydrodynamic theory calculation, but the power loss for such a water transport in each cellular connexon channel is still very small as on the scale no more than 1.0E-9Watt.

\section{REFERENCES}

Borg, M. K., Reese, J. M., 2017, "Multiscale simulation of enhanced water flow in nanotubes."MRS Bulletin, 42, 294-299. https://doi.org/10.1557/mrs.2017.59

Holt, J. K., Park, H. G., Wang, Y., Stadermann, M., Artyukhin, A. B., Grigoropoulos, C. P., Noy, A., Bakajin, O., 2006, "Fast mass transport through sub-2-nanometer carbon nanotubes.” Science, 312, 1034-1037. https://doi.org/10.1126/science.1126298

Jiang, C. T., Zhang, Y. B., 2021, "Direct matching between the flow factor approach model and molecular dynamics simulation for nanochannel flows." Int. Commun. Heat Mass Transf., submitted.

Khademi, M., Sahimi, M., 2011, "Molecular dynamics simulation of pressure-driven water flow in silicon-carbide nanotubes." J. Chem. Phys. 135, 204509.

https://doi.org/10.1063/1.3663620

Li, Z. X.,2010, "Experimental study on flow characteristics of liquid in circular microtubes." Microscale Thermophys. Eng., 7, 253-265. https://doi.org/10.1080/10893950390219083

Li, J., Zhang, Y. B., 2021, "Flow equations and their borderlines for different regimes of mass transfer." Front Heat Mass Transf., 16, 21. http://dx.doi.org/10.5098/hmt.16.21

Majumder, M., Chopra, N., Andrews, R., Hinds, B. J., 2005, "Enhanced flow in carbon nanotubes." Nature, 438, 44.

https://doi.org/10.1038/438044a

Thomas, J. A., McGaughey, A. J. H., Kuter-Arnebeck, O., 2010, "Pressure-driven water flow through carbon nanotubes: Insights from molecular dynamics simulation." Int. J. Thermal Sci., 49, 281-289. https://doi.org/10.1016/j.ijthermalsci.2009.07.008

Walther, J. H., Praprotnik, M. J., Kotsalis, E. M., Koumoutsakos, P., 2012, "Multiscale simulation of water flow past a C540 fullerene." $J$. Comput. Phys., 231, 2677-2681. https://doi.org/10.1016/i.jcp.2011.12.015

Wang, L., Dumont, R. S., Dickson, J. M., 2012, "Nonequilibrium molecular dynamics simulation of water transport through carbon nanotube membranes at low pressure.” J. Chem. Phys. 137, 044102. https://doi.org/10.1063/1.4734484

Whitby, M., Cagnon, L., Thanou, M., Quirke, N., 2008, "Enhanced fluid flow through nanoscale carbon pipes." Nano Lett., 8, 2632-2637. https://doi.org/10.1021/n1080705f

Zhang, Y. B., 2006, "Flow factor of non-continuum fluids in onedimensional contact." Industr. Lubr. Trib., 58, 151-169.

http://dx.doi.org/10.1108/00368790610661999 
Zhang, Y. B., 2014, "Lubrication analysis for a line contact covering from boundary lubrication to hydrodynamic lubrication: Part I- Micro contact results." J. Comput. Theor. Nanosci., 11, 62-70. https://doi.org/10.1166/jetn.2014.3318

Zhang, Y. B., 2016, "The flow equation for a nanoscale fluid flow." Int. J. Heat Mass Transf., 92, 1004-1008.

https://doi.org/10.1016/j.ijheatmasstransfer.2015.09.008
Zhang, Y. B., 2017, "Transport in nanotube tree." Int. J. Heat Mass Transf., 114, 536-540.

http://dx.doi.org/10.1016/j.ijheatmasstransfer.2017.06.105

Zhang, Y. B., 2020, "Modeling of flow in a micro cylindrical tube with the adsorbed layer effect: Part I-Results for no interfacial slippage." Int. J. Heat Mass Transf., submitted. 